\title{
Identifying Risk Factors for Levels of Per- and Polyfluoroalkyl Substances (PFAS) in the Placenta in a High-Risk Pregnancy Cohort in North Carolina
}

\author{
Jacqueline Bangma, Lauren A. Eaves, Kirsi Oldenburg, Jessica L. Reiner, Tracy Manuck, \\ and Rebecca C. Fry*
}

\begin{abstract}
Prenatal exposure to per- and polyfluoroalkyl substances (PFAS), a ubiquitous class of chemicals, is associated with adverse outcomes such as pre-eclampsia, low infant birth weight, and later-life adiposity. The objectives of this study were to examine PFAS levels in the placenta and identify sociodemographic risk factors in a high-risk pregnancy cohort $(n=122)$ in Chapel Hill, North Carolina. Of concern, PFOS, PFHxS, PFHpS, and PFUnA were detected above the reporting limit in 99, 75, 55, and $49 \%$ of placentas, respectively. Maternal race/ethnicity was associated with significant differences in PFUnA levels. While the data from this high-risk cohort did not provide evidence for an association with hypertensive disorders of pregnancy, fetal growth,
\end{abstract} or gestational age, the prevalence of detectable PFAS in the

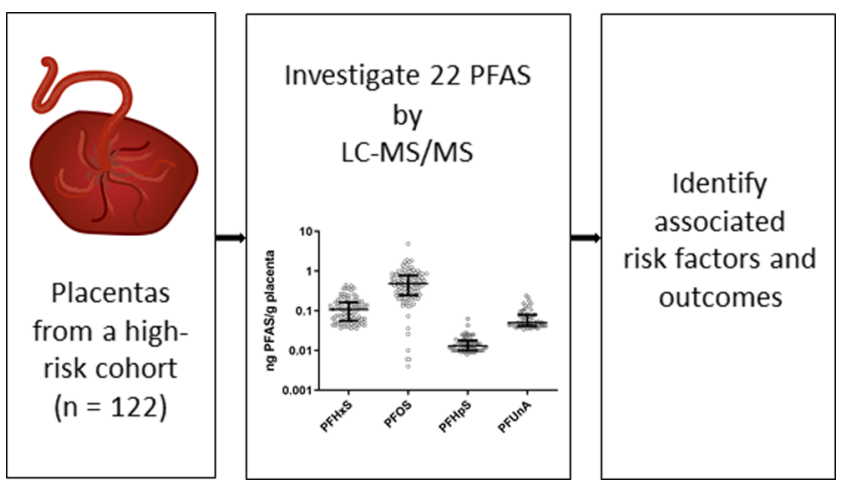
placenta suggests a need to biomonitor for exposure to PFAS during pregnancy. Future research should investigate factors underlying the differences in PFAS levels in association with a mother's race/ethnicity, as well as potential effects on pregnancy and child health.

\section{INTRODUCTION}

Per- and polyfluoroalkyl acids (PFAS) are a diverse chemical family of synthetic organic structures used for a variety of applications in both the United States and worldwide due to their water- and grease-resistant properties. ${ }^{1}$ PFAS are found in fabric surface treatment, pesticides, fire-fighting foam, food packaging, and a variety of other sources, reaching humans via air, contaminated water, food, and breast milk. ${ }^{2-8}$ PFAS consist of alkyl chains in which hydrogen atoms have been replaced by fluorine. These fluorine-hydrogen bonds are exceptionally strong, making PFAS resistant to degradation and highly persistent in the environment and humans. ${ }^{9}$ Due to their persistence and pervasive use, PFAS have been detected in human blood worldwide. ${ }^{10,11}$ Between 1999 and 2012, a number of PFAS were found at detectable levels in blood in over $98 \%$ of the U.S. population. ${ }^{12,13}$ Concern is growing regarding widespread exposure to PFAS, and a number of PFAS are currently listed as Contaminants of Emerging Concern (CECs) by the U.S. Environmental Protection Agency. ${ }^{14}$

PFAS exposure in pregnant women has been associated with an increased risk of fertility problems, pre-eclampsia, and miscarriage. ${ }^{15-21}$ In utero PFAS exposure has been associated with an array of negative effects in children: low infant birth weight, ${ }^{22-27}$ congenital cerebral palsy in boys, ${ }^{28}$ dysregulated pubertal onset later in life, ${ }^{29}$ and greater adiposity. ${ }^{30}$ Animal models have also shown deleterious effects of exposure to PFAS, including altered pubertal onset, reduced postnatal survival, and disturbed lactation in rodents exposed in utero. ${ }^{31-34}$ While a number of studies have focused on pregnancy as a time window of exposure to PFAS by assessing maternal blood or serum, few have focused on the placenta that is critical for understanding the effect of PFAS during pregnancy. ${ }^{35,36}$ As the transducer of the maternal to fetal environment during pregnancy, the placenta is important for adequate transport of nutrients, removal of waste, and secretion of hormones. Studies have shown that PFAS can transfer between maternal and fetal circulation via the placenta. ${ }^{37-40}$ Additionally, the placenta:maternal serum ratios of PFAS have been found to increase with gestational age, suggestive of the bioaccumulation of PFAS in the placenta over time. ${ }^{41}$ Despite growing concern over the effects of PFAS on pregnancy, fetal development, and their ability to cross the placenta, only three studies to date, all of

Received: November 22, 2019

Revised: $\quad$ May 28, 2020

Accepted: May 29, 2020

Published: May 29, 2020 
which studied women outside of the United States, have detailed PFAS levels in the placenta. ${ }^{40-42}$ This study aimed to address this research gap in understanding the levels of PFAS in the placentas of a U.S.-based cohort and identify risk factors associated with high exposure.

In addition, the majority of studies on PFAS exposure focus on two perfluoroalkyl acids, mainly perfluorooctane sulfonate (PFOS) and perfluorooctanoic acid (PFOA); ${ }^{43}$ however, over 3000 PFAS are estimated to be in production. ${ }^{1}$ While some of these chemicals, including PFOS and PFOA, have been discontinued by major manufacturers in the United States, their persistence in the environment and continued use internationally make them a continued toxicological concern. ${ }^{44}$ Moreover, hexafluoropropylene oxide dimer acid (HFPO-DA, ammonium salt with trade name: GenX), a modern replacement for PFOA, has recently contaminated drinking water from the Cape Fear River in the Wilmington area, NC, warranting immediate analysis of the prevalence of PFAS in NC populations. $^{45}$

In the present study, we set out to characterize the PFAS level in the placenta and understand risk factors associated with high PFAS exposure in a high-risk pregnancy cohort in the United States. To carry out this research, we utilized specimens from a cohort of pregnant women at risk for preterm birth in North Carolina. The primary goals of this work were to (i) establish baseline data on 22 different PFAS levels in the placenta, (ii) identify sociodemographic risk factors associated with increased placental PFAS levels in this cohort, and (iii) investigate whether PFAS placental levels change with gestational age at delivery, fetal growth, or hypertensive disorders of pregnancy (preeclampsia and gestational hypertension).

\section{MATERIALS AND METHODS}

2.1. Study Population. This cross-sectional study was derived from the UNC Preterm Birth Biobank (PTB) Study. ${ }^{46}$ The original purpose of this overarching study was to establish a database and tissue bank to evaluate clinical and biochemical markers of preterm birth. Participants were identified and recruited through UNC-CH obstetrics and gynecology clinics and inpatient obstetric units at the North Carolina Women's Hospital from 2015 to 2018. Women at high risk for spontaneous preterm birth due to (a) a prior spontaneous preterm birth, (b) short mid-trimester transvaginal cervical length ( $<25 \mathrm{~mm}$, assessed between 16.0 and 23.9 weeks' gestation) in the current pregnancy, (c) twin or triplet gestation, or (d) antepartum admission due to threatened preterm labor following cervical change and/or cervical dilation in the setting of at least six symptomatic uterine contractions per hour were included in the UNC PTB Biobank. Women carrying fetuses with major structural anomalies or aneuploidy were excluded. Pregnancies were dated by the last menstrual period (if available) and ultrasound using standard American College of Obstetricians and Gynecologists criteria. ${ }^{47}$ All pregnancy management decisions were made at the discretion of the primary obstetric provider. This study was approved by the Institutional Review Board of the University of North Carolina, Chapel Hill (IRB\# 14-2855), and all women provided written, informed consent.

Baseline clinical and demographic information (including maternal educational attainment, race and ethnicity, marital status, and prepregnancy body mass index $\left(\mathrm{kg} / \mathrm{m}^{2}\right)$ ) were collected from participants through structured interviews and supplemented by a review of electronic medical records. In addition, the antenatal course, delivery indications, and pregnancy outcomes (including infant gender, birth weight, gestational age at delivery) were obtained from the electronic medical record following delivery.

After delivery, three full-thickness placental biopsies were obtained from the fetal side, $2 \mathrm{~cm}$ from the placental cord insertion site using a standard $3 \mathrm{~mm}$ punch biopsy tool, and frozen at $-80{ }^{\circ} \mathrm{C}$ within $24 \mathrm{~h}$ of delivery until analysis. Care was taken to avoid biopsies of placental sites with obvious gross abnormalities. Two hundred and seventy-one women were enrolled in the UNC PTB Biobank study: 2 had a first-trimester miscarriage, 13 were lost to follow-up or had missing data on gestational age at delivery, resulting in 256 viable placentas, of which 122 had enough remaining placental sample to be used in the present analysis.

2.2. Placenta PFAS Quantification. Extraction and quantification by liquid chromatography-mass spectrometry (LC-MS/MS) method established utilized in this study were previously established in Bangma et al. 2018 and included 15 total PFAS. ${ }^{48}$ In the current study, the method was updated to include a total of 22 PFAS as follows: perfluorobutyric acid (PFBA), perfluoropentanoic acid (PFPeA), perfluorohexanoic acid (PFHxA), perfluoroheptanoic acid (PFHpA), perfluorooctanic acid (PFOA), perfluorononanoic acid (PFNA), perfluorodecanoic acid (PFDA), perfluoroundecanoic acid (PFUdA), perfluorododecanoic acid (PFDoA), perfluorotridecanoic acid (PFTrDA), perfluorotetradecanoic acid (PFTeDA), perfluorohexadecanoic acid (PFHxDA), perfluorobutanesulfonic acid (PFBS), perfluoropentanesulfonic acid (PFPeS), perfluorohexanesulfonic acid (PFHxS), perfluoroheptanesulfonic acid (PFHpS), perfluorooctane sulfonic acid (PFOS), perfluorononanesulfonic acid (PFNS), perfluorodecanesulfonic acid (PFDS), perfluorododecancesulfonic acid (PFDoS), $1 \mathrm{H}, 1 \mathrm{H}, 2 \mathrm{H}, 2 \mathrm{H}$-perfluorooctane sulfonic acid (6:2 FTS), and 2,3,3,3-tetrafluoro-2-(1,1,2,2,3,3,3-heptafluoropropoxy)propanoic acid (HFPO-DA also known as GenX). In-depth information detailing the Bangma et al. 2018 method as it was utilized for placenta PFAS quantification can be found in the Supporting Information (SI).

2.3. Statistical Analysis. PFHpS, PFHxS, PFOS, and PFUnA were identified as having approximately $50 \%$ or more of samples over the reporting limit (RL). Using an established gravimetric method, the reporting limit $(\mathrm{RL})$ is calculated in two ways: (1) using the lowest detectable calibrant divided by the mass of the extracted sample or (2) taking the average amount of compound detected in the blanks divided by the mass of the extracted sample. ${ }^{49,50}$ To be conservative, from the two methods of calculating the RL, the RL that was the highest for that PFAS and sample was designated as the respective RL for PFAS in that sample (Supporting Information File S1). The concentrations determined include isomers for applicable PFAS. For statistical analysis, all samples below the RL were set to half the $R L$ detection limit for that sample and PFAS. ${ }^{51}$ This allowed for the preservation of sample size for the PFAS included in the statistical analysis. Spearman rank correlations between concentrations of each of the PFAS were calculated to evaluate patterns of co-occurrence.

For this analysis, the race was categorized as non-hispanic white, hispanic white, non-hispanic black, or other. Education was trichotomized into less than high school, completed high school, or further education beyond high school. Marital status was dichotomized as married or not married. Delivery gestational age was maintained as a continuous variable for 
Table 1. Study Population Characteristics $(N=122)$ of Women with Placental Samples Analyzed for PFAS Levels (Chapel Hill, NC)

\begin{tabular}{|c|c|c|c|c|c|c|c|c|c|c|c|}
\hline & range & $\begin{array}{l}\text { mean } \\
(\mathrm{SD})\end{array}$ & $N$ & $(\%)^{a}$ & $\begin{array}{l}\text { missing } \\
\text { values } \\
(\%)^{b}\end{array}$ & & range & $\begin{array}{l}\text { mean } \\
(\mathrm{SD})\end{array}$ & $N$ & $(\%)^{a}$ & $\begin{array}{l}\text { missing } \\
\text { values } \\
(\%)^{b}\end{array}$ \\
\hline race and hispanic & & & & & $10(8.2)$ & less than high school & & & 13 & $(12.8)$ & \\
\hline & & & & & & completed high school & & & 42 & $(41.2)$ & \\
\hline white, non-hispanic & & & 42 & $(37.5)$ & & more than high school & & & 34 & $(33.3)$ & \\
\hline white, hispanic & & & 30 & $(26.8)$ & & declined & & & 13 & $(12.8)$ & \\
\hline black, non-hispanic & & & 37 & $(33.0)$ & & marital status & & & & & $8(6.6)$ \\
\hline other & & & 3 & $(2.7)$ & & married & & & 81 & $(71.0)$ & \\
\hline maternal smoking & & & & & $1(<1)$ & not married & & & 33 & $(29.0)$ & \\
\hline yes & & & 9 & $(7.4)$ & & gestational age (weeks) & $18-41$ & 33.9 & & & $1(<1)$ \\
\hline no & & & 112 & $(92.6)$ & & & & $(5.55)$ & & & \\
\hline child's gender & & & & & $1(<1)$ & preterm delivery & & & & & \\
\hline female & & & 54 & $(44.6)$ & & very preterm $(<34$ & & & 44 & $(36.4)$ & \\
\hline male & & & 67 & $(55.4)$ & & weeks) & & & & & \\
\hline $\begin{array}{l}\text { maternal prepregnancy } \\
\text { BMI }\left(\mathrm{kg} / \mathrm{m}^{2}\right)\end{array}$ & $17-56$ & $\begin{array}{r}30.9 \\
(8.82)\end{array}$ & & & 0 & $\begin{array}{l}\text { preterm } \\
\quad(34 \leq \text { weeks }<37)\end{array}$ & & & 12 & $(9.9)$ & \\
\hline$<18.5$ & & & 6 & $(4.9)$ & & term ( $\geq 37$ weeks) & & & 65 & $(53.7)$ & \\
\hline $18.5 \leq 25$ & & & 24 & $(19.7)$ & & hypertensive & & & & & $1(<1)$ \\
\hline $25 \leq 30$ & & & 30 & $(24.6)$ & & $\begin{array}{l}\text { complications of } \\
\text { pregnancy }\end{array}$ & & & & & \\
\hline $30+$ & & & 62 & $(50.8)$ & & no hypertensive & & & 107 & $(88.4)$ & \\
\hline maternal age & $20-44$ & 31.0 & & & $1(<1)$ & complications & & & & & \\
\hline $\begin{array}{l}\text { below } 20 \text { years old } \\
\quad(<20)\end{array}$ & & $(6.51)$ & 0 & (0) & & $\begin{array}{l}\text { pre-eclampsia and/or } \\
\text { gestational } \\
\text { hypertension }\end{array}$ & & & 14 & $(11.6)$ & \\
\hline $\begin{array}{l}\text { between } 20 \text { and } 25 \text { years } \\
\text { old }(\geq 20 \text { to }<35)\end{array}$ & & & 77 & $(63.1)$ & & birth weight (grams) & $159-4300$ & $\begin{array}{l}2375 \\
(1085)\end{array}$ & & & $5(4.1)$ \\
\hline over 35 years old $(\geq 35)$ & & & 44 & $(36.1)$ & & small for gestational age & & & & & $9(7.4)$ \\
\hline $\begin{array}{l}\text { maternal medical } \\
\text { insurance }\end{array}$ & & & & & ${ }^{18}(14.8)$ & $\begin{array}{l}\text { (SGA)/large for } \\
\text { gestational age (LGA) }\end{array}$ & & & & & \\
\hline private insurance & & & 56 & $(53.9)$ & & SGA & & & 9 & 8.0 & \\
\hline Medicare/Medicaid & & & 40 & $(38.5)$ & & AGA & & & 92 & 81.4 & \\
\hline self-pay & & & 8 & $(7.7)$ & & LGA & & & 12 & 10.6 & \\
\hline maternal education & & & & & ${ }^{20}(16.4)$ & ${ }^{a}$ Percent of nonmissi & observati & s. ${ }^{b}$ Perce & it of & observ & tions. \\
\hline
\end{tabular}

regression analyses and was also trichotomized into very preterm (delivery before $34^{0 / 7}$ weeks of gestation), preterm (delivery between $34^{0 / 7}$ and $36^{6 / 7}$ weeks of gestation), and term (delivery $\geq 37^{0 / 7}$ weeks of gestation) for a categorical variable. Insurance was trichotomized into private insurance, Medicaid/Medicare insurance, or self-pay. Maternal age was trichotomized into below 20 years old at delivery ( $<20$ years), between 20 and 35 years of age at delivery ( $\geq 20$ to $<35$ years), and over 35 years and older at delivery ( $\geq 35$ years). Prepregnancy BMI was categorized into underweight (BMI $<18.5 \mathrm{~kg} / \mathrm{m}^{2}$ ), normal weight $\left(\geq 18.5-24.9 \mathrm{~kg} / \mathrm{m}^{2}\right)$, overweight $\left(\geq 25.0-29.9 \mathrm{~kg} / \mathrm{m}^{2}\right)$, and obese $\left(\geq 30.0 \mathrm{~kg} / \mathrm{m}^{2}\right)$ categories. Gestational hypertension was defined as new-onset hypertension, with at least two blood pressure readings $\geq 140 / 90$ at least 6 h apart at or after 20 weeks' gestation. Pre-eclampsia was defined as hypertension accompanied by new-onset proteinuria (urine protein:creatine ratio $\geq 0.30$ or $24 \mathrm{~h}$ urine protein $\geq 300 \mathrm{mg} / \mathrm{dL}$ ) and/or laboratory abnormalities (platelet count $<100$ per microliter, serum aspartate transaminase, or aminotransferase twice normal or $\geq 70 \mathrm{U} / \mathrm{L}$ ) and/or severe headache or visual disturbance. Women with chronic hypertension were considered to have super-imposed pre-eclampsia if they had an exacerbation of their hypertension in addition to one of the above laboratory abnormalities or physical symptoms. Because the American College of Obstetricians and Gynecologists recognize that preeclampsia can develop in the absence of proteinuria, and the management of gestational hypertension and pre-eclampsia is

similar, these diagnoses were combined into one "hypertensive disorders of pregnancy" variable, dichotomized as yes or no. ${ }^{47}$ For assessing the association with fetal growth, small for gestational age (SGA), appropriate for gestational age (AGA), and large for gestational age (LGA) newborns were identified using gender-specific fetal growth curves from a nationally representative, racially diverse reference population. Small for gestational age was defined as birth weight in grams $\leq 10$ th percentile and large for gestational age was defined as birth weight in grams $\geq 90$ th percentile of this reference population. ${ }^{52}$ Those born $<23$ weeks' gestation were considered as having missing data for fetal growth category because there is no nationally representative data on birth weight distributions below this gestational age from the reference used. Smoking status was not included in analyses as only 9 mothers reported active smoking during pregnancy and 112 mothers reported nonsmoking status.

Distributions of each PFAS were evaluated for normality via Shapiro-Wilks tests of both original and log-transformed data. All PFAS evaluated were determined to have non-normal distributions in both original and log-transformed forms; thus, to examine differences across categorical variables, nonparametric tests were employed to test the equality of medians. Wilcoxon tests were used to evaluate differences between dichotomous sociodemographic variables and hypertensive disorders of pregnancy, and Kruskal-Wallis tests were used to evaluate differences between sociodemographic variables with 
Table 2. Of 22 Monitored PFAS, 11 That Were Detected in PTB Biobank Placenta $(n=122)$ Are Shown Here ${ }^{a, b}$

\begin{tabular}{|c|c|c|c|c|c|c|c|c|c|c|c|}
\hline & PFPeS & $\mathrm{PFHxS}^{c}$ & $\mathrm{PFHpS}^{c}$ & PFOS $^{c}$ & PFHxA & PFOA & PFNA & PFDA & PFUnA $^{c}$ & PFTriA & PFTA \\
\hline chain length & 5 & 6 & 7 & 8 & 6 & 8 & 9 & 10 & 11 & 13 & 14 \\
\hline$n$ & 122 & 122 & 122 & 122 & 122 & 122 & 122 & 122 & 122 & 122 & 122 \\
\hline$\%>\mathrm{RL}$ & 31 & 75 & 55 & 99 & 1.6 & 27 & 21 & 39 & 49 & 29 & 7 \\
\hline maximum (ng/g) & 0.035 & 0.446 & 0.063 & 4.87 & 5.87 & 1.23 & 0.494 & 0.465 & 0.240 & 0.336 & 0.111 \\
\hline minimum $(\mathrm{ng} / \mathrm{g})$ & $<0.005$ & $<0.033$ & $<0.008$ & $<0.001$ & $<1.32$ & $<0.290$ & $<0.148$ & $<0.030$ & $<0.033$ & $<0.050$ & $<0.049$ \\
\hline median (ng/g) & $<0.005$ & 0.067 & 0.009 & 0.480 & $<1.45$ & $<0.315$ & $<0.163$ & $<0.031$ & $<0.031$ & $<0.057$ & $<0.054$ \\
\hline
\end{tabular}

${ }^{a}$ PFAS with approximately $50 \%$ or more of samples above the reporting limit (RL) were included in the statistical analysis. ${ }^{b}$ Values below the RL are listed in this table as " $\angle \mathrm{RL}$ ". ${ }^{c}$ Included in the statistical analysis.

more than two groups and fetal growth categories. The $p$-values of these tests are reported with significance defined as $p<0.05$.

To evaluate whether PFAS levels varied by gestational age, both univariate and multivariate linear regression models were performed to generate crude and adjusted estimates of change in PFAS levels by gestational age. For the multivariate linear regression, control variables were identified as having significant or borderline significant differences in medians across groups from the sociodemographic risk factor analysis described above. To assess temporal changes in PFAS concentrations of the placenta, univariate linear regression models were used to evaluate the association between delivery date and PFAS concentration. For this model, births in 2018 were excluded due to a low sample size $(n=2$ births in 2018). All analyses were conducted in SAS 9.4 software.

\section{RESULTS}

3.1. Study Population Characteristics. This cohort of 122 women included $42(37.5 \%)$ non-hispanic white, 30 (26.8\%) hispanic white, 37 (33.0\%) non-hispanic black, and 3 (2.7\%) women who self-reported other for race/ethnicity (Table 1$)$. The majority (63.1\%) of women were between 20 and 34 years old. Just over half (53.9\%) had private medical insurance, with $38.5 \%$ using Medicaid/Medicare insurance. In this high-risk group, the mean gestational age at delivery was 33.9 weeks. A total of 44 women (36.9\%) delivered very preterm (less than 34 weeks), 12 women (9.8\%) delivered preterm (between 34 and 37 weeks), and 65 women (53.3\%) delivered term (37 weeks or over). Fourteen (11.5\%) women had pregnancies complicated by hypertensive disorders. The mean birth weight was $2,375 \mathrm{~g}$ (range $159-4300 \mathrm{~g}$ ). In comparison to the National Vital Statistics data from 2016 and 2017 representatives of all births in the United States, the present cohort differs slightly with regard to racial demographics (U.S. population, 2017: $51.68 \%$ white mothers, $14.54 \%$ to nonhispanic black, $23.3 \%$ hispanic) but is similar with regard to the percentage of insurance types (U.S. population, 2017: 49.1\% private insurance, $43 \%$ Medicaid). ${ }^{53,54}$ As expected, given the high-risk characteristics of the cohort, the mean gestational age at delivery (prevalence of preterm births) and mean birth weight differed from the general U.S. population. ${ }^{53,54}$

3.2. PFAS Levels in the Study Population. Of the 22 PFAS examined in the placenta, 11 PFAS were detected in at least one placenta sample $(n=122)$ (Table 2). Compared to three other non-U.S.-based studies of placenta PFAS levels, these data are relatively consistent, with PFOS and PFOA found at the maximum values (Figure 1). PFHxS, PFHpS, PFOS, and PFUnA were detected over the RL in approximately $50 \%$ or more of samples. As a significant lack of detection would skew results, for all subsequent analyses, only these four PFAS are considered and values below the $\mathrm{RL}$ were imputed as half the
RL. The highest levels detected were of PFOS, followed by PFHxS, PFUnA, and then PFHpS (Figure 2). PFOS was detected at a median of $0.480 \mathrm{ng} / \mathrm{g}$ and a maximum of $4.87 \mathrm{ng} / \mathrm{g}$. Comparatively, PFHxS had a median of $0.067 \mathrm{ng} / \mathrm{g}$ and a maximum of $0.446 \mathrm{ng} / \mathrm{g}$ and PFUnA had a median value under the $\mathrm{RL}$ and a maximum of $0.240 \mathrm{ng} / \mathrm{g}$. PFHpS was found to have a median concentration of $0.009 \mathrm{ng} / \mathrm{g}$ and a maximum of 0.063 $\mathrm{ng} / \mathrm{g}$. Correlation between the levels of PFHxS, PFHpS, PFOS, and PFUnA was common (Supporting Information Figure S1 and Table S1). Specifically, all pairings other than PFUnA and PFHxS were significantly positively correlated. The most significant correlation was PFHpS and PFHxS (Spearman correlation coefficient $=0.532$, $p$-value $\left.=2.907 \times 10^{-10}\right)$.

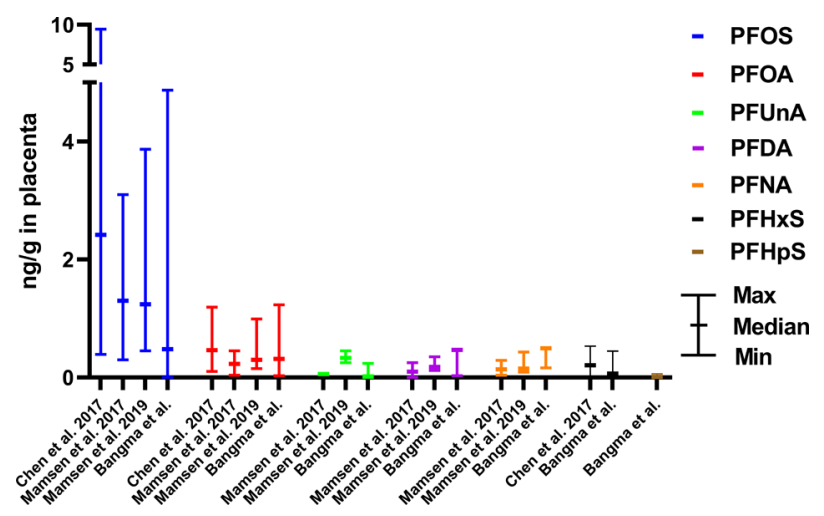

Figure 1. Maximum, minimum, and median (means are indicated when medians were not reported in the literature) PFAS levels measured by $\mathrm{ng} / \mathrm{g}$ wet weight in the human placenta as reported in the literature for Chen et al. $2017(n=32)$, Mamsen et al. $2019(n=78)$, Mamsen et al. 2017 ( $n=34$, means reported), and placentas included in the current study, Bangma et al. $(n=122)$. Frequently reported PFAS, including PFOS, PFOA, PFNA, and PFDA, were included in the graph as well as any additional PFAS detected in $\sim 50 \%$ of the placenta in the current study, including PFUnA, PFHxS, and PFHpS.

The median and interquartile ranges of PFAS in the total cohort and stratified by select sociodemographic characteristics were calculated (Table 3). Median and interquartile ranges for the remaining sociodemographic characteristics can be found in the Supporting Information Table S2. Additionally, $p$-value results of Wilcoxon or Kruskal-Wallis test for difference in median levels are displayed. Two comparisons were statistically significant $(p<0.05)$. PFUnA levels in the placenta were significantly different according to race/ethnicity status ( $p=$ $0.0002)$. One comparison was approaching the statistical significance: difference in PFHpS levels in the placenta in mothers of different ages $(p=0.0936)$.

3.3. PFAS Levels and Race/Ethnicity. Significant differences in PFUnA levels were observed by maternal race and 


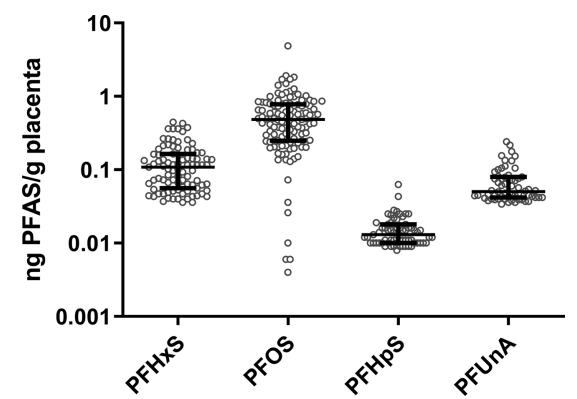

Figure 2. Whisker plots of the distribution of PFAS of the four PFAS included in statistical analysis: PFHpS, PFHxS, PFOS, and PFUnA. Circles represent individual PFAS values, and bars represent median and interquartile ranges.

ethnicity. The three women of other race/ethnicities had the highest maximum and median values: 0.156 and $0.081 \mathrm{ng} / \mathrm{g}$, respectively. This was followed by hispanic white, non-hispanic white, and non-hispanic black women, according to their median value. No other comparisons of PFAS levels by race/ethnicity were significant, and the order from the highest to lowest median values was not consistent across the different PFAS.

3.4. PFAS Levels and Maternal Age. Differences by maternal age in PFHpS were trending toward significance $(p=$ 0.09 ). Older mothers were observed to have higher levels of PFHpS than younger mothers. The median PFHpS in mothers under 20 years old was $0.004 \mathrm{ng} / \mathrm{g}$, mothers between 20 and 35 years old had a median level of $0.009 \mathrm{ng} / \mathrm{g}$, and mothers over the age of 35 had a median level of $0.011 \mathrm{ng} / \mathrm{g}$.

3.5. PFAS Levels and Hypertensive Complications of Pregnancy. No significant differences in PFHxS, PFHpS, or PFOS by hypertensive disorders of pregnancy were found (Supporting Information Table S3). A borderline significant difference was observed of PFUnA levels by hypertensive complications of pregnancy status $(p=0.09)$. In women with hypertensive complications of pregnancy, the median PFUnA level was $0.044 \mathrm{ng} / \mathrm{g}$, compared to $0.020 \mathrm{ng} / \mathrm{g}$ in women without hypertensive complications of pregnancy. All median values were higher in placentas of mothers with hypertensive complications of pregnancy. Associations were examined in a sex-specific manner (e.g., stratified by fetal sex), and no significant differences were observed (data not shown).

3.6. PFAS Levels and Fetal Growth. Fetal growth was categorized into small for gestational age (SGA), appropriate for gestational age (AGA), and large for gestational (LGA). Of the cohorts, $8.0 \%$ was SGA and $10.6 \%$ was LGA. No significant differences in median placental levels of PFOS, PFHxS, PFHpS, nor PFUnA were seen by fetal growth categories (Supporting Information Table S4). No consistent patterns were seen for PFAS levels across fetal growth categories. Associations were examined in a sex-specific manner (e.g., stratified by fetal sex), and no significant differences were observed (data not shown).

3.7. PFAS Levels and Gestational Age. Gestational ages in this cohort ranged from 18 to 41 weeks. Both crude and adjusted linear regression models regressing PFAS level onto gestational age showed no significant association (Supporting Information Table S5A,B). $\beta$ values representing the predicted change in the PFAS level in $\mu \mathrm{g} / \mathrm{g}$ with each additional week of gestation were positive for PFHxS and PFHpS (increase in the placental PFAS level with an increase in gestational age) and negative for PFOS and PFUnA (a decrease in the placental

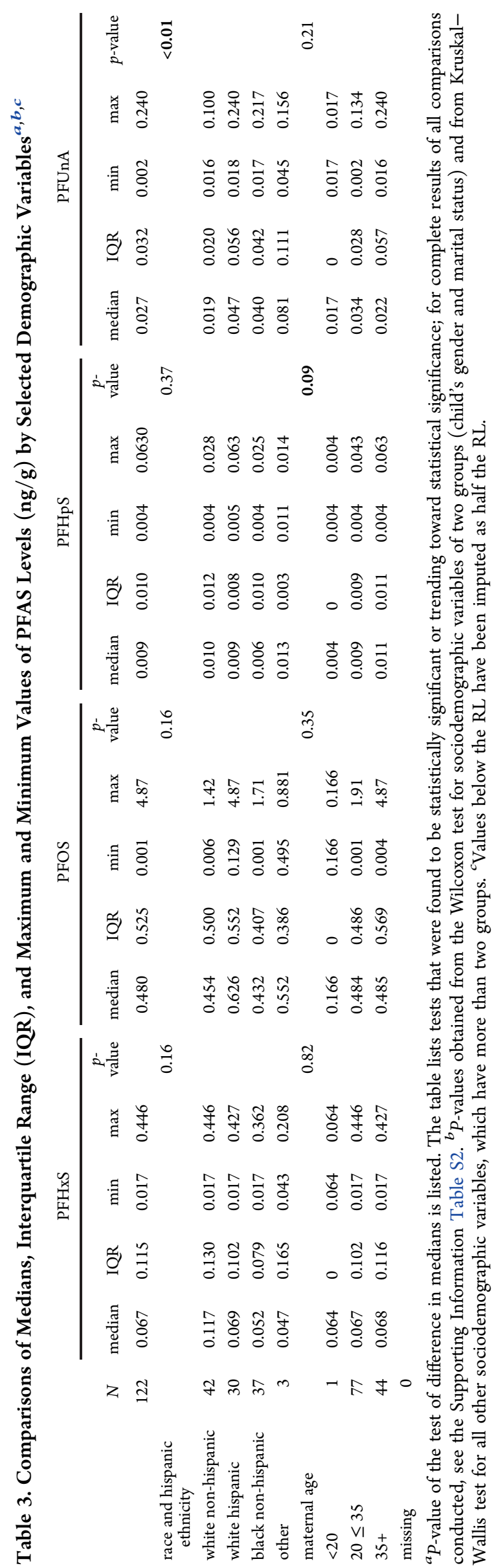


PFAS level with an increase in gestational age) in both the crude and adjusted models.

For the remaining seven PFAS detected below 50\% RL, associations between gestational age and detection of PFAS above the RL were investigated utilizing unadjusted binomial regression models. PFAS levels were dichotomized into two categories (detected above RL or detected below RL). PFOA was significantly more likely $(p=0.04)$ to be detected above RL in later gestational ages compared to that in earlier gestation age placentas (Supporting Information Table S6). Associations were examined in a sex-specific manner (e.g., stratified by fetal sex), and no significant differences were observed (data not shown).

3.8. PFAS Levels Over Time (Year of Delivery). Dates of birth in this cohort ranged from $04 / 12 / 2015$ to $01 / 12 / 2018$. As only two participants had births in 2018 , for the analysis of the effect of year of delivery, these two observations were removed. PFOS, PFHxS, and PFUnA were all found to decrease in concentration in placental tissues over time (Supporting Information Table S7). However, only PFHxS significantly decreased over time, with a predicted change with each passing year of $-0.024 \mathrm{ng} / \mathrm{g}(p$-value $=0.04)$. PFHpS showed an increase in levels with each subsequent year; however, this association was not significant.

\section{DISCUSSION}

PFAS exposure during pregnancy has been associated with health risks for both the mother and the fetus. ${ }^{15,16,20,22,26,27,30,55}$ Despite growing concern over the effects of prenatal exposure to PFAS, and the understanding that PFAS can cross the maternal fetal barrier via the placenta, a limited number of studies have evaluated PFAS levels in the placenta. ${ }^{37-40}$ Investigating the placenta separately from maternal serum can lend additional information as to the extent to which PFAS accumulate in the placenta. This is particularly important because it cannot be assumed that serum concentrations are representative of PFAS accumulation in specific critical organs. For example, studies have observed certain PFAS in higher levels in brain and lung compartments despite low rates of detection in serum. ${ }^{56}$ The present study provides novel information as it is among the first to quantify and analyze placental levels of PFAS in a U.S. cohort. Overall, there were three major findings. First, many of the placental samples had levels of specific PFAS chemicals similar to international studies. Of concern, PFOS, PFHxS, PFHpS, and PFUnA were detected above the reporting limit in 99, 75, 55, and $49 \%$ of placentas, respectively. Second, placental PFAS levels varied by race/ethnicity for PFUnA. Finally, associations between the levels of PFAS in the placenta in the study cohort and gestational age, hypertensive disorders of pregnancy, and fetal growth were performed but did not reach statistical significance. While placental PFAS were not associated with pregnancy or child health outcomes in this high-risk cohort, the prevalence of their detection in the placenta suggests a need to biomonitor during pregnancy.

Of the four PFAS detected above RL in approximately $50 \%$ or more of samples, PFOS was found in the highest concentration. This finding is consistent with the current literature. Previously published studies on PFAS in the placenta are limited to three studies: one in China and two in Denmark. ${ }^{40-42}$ Similar to the results from the present study, these studies found PFOS and PFOA as having the highest and second highest median ng/g wet weights, respectively, compared to other PFAS. Moreover, in the analysis of PFAS in serum measured by the National Health and Nutrition Examination Survey (NHANES) from
1999 to 2016, PFOS was consistently found in the greatest concentration compared to other PFAS. ${ }^{57}$ Notably, of the four PFAS detected above RL in the present study, only PFOS and PFHxS are currently tracked by NHANES; therefore, comparison to the general US population for exposure to PFHpS and PFUnA is not possible at this time. In NHANES, the geometric means in the serum of PFOS and PFHxS (20152016) are 4.72 and $1.18 \mu \mathrm{g} / \mathrm{L}$, respectively. ${ }^{57}$ Despite PFOS being phased out of production in the United States since $2002,{ }^{57,58}$ the presence of PFOS in the placenta in this study highlights the persistence of PFAS and signifies the importance of continued PFAS monitoring and reduction efforts.

In the present study, maternal race and ethnicity were associated with significant differences in PFUnA levels, with those who self-reported their race/ethnicity as other than nonhispanic white, hispanic white, and non-hispanic black having the greatest measured median wet weight in ng/g of PFUnA, while non-hispanic black women had the lowest. PFAS concentrations have been found to vary by race/ethnicity in nonpregnancy cohorts; however, consistent patterns have not been established and different PFAS appear to demonstrate varied distributions by race and ethnicity. ${ }^{59-61}$ Research concerning associations between race/ethnicity and PFAS levels in pregnant women is limited. To our knowledge, the only studies to investigate race/ethnicity and PFAS in pregnant U.S. women are the Health Outcomes and Measures of the Environment (HOME) Study and Project Viva. The HOME study observed significantly different serum PFAS concentrations in pregnant mothers based on maternal race. ${ }^{36,62,63}$ In addition, both Project Viva and the HOME Study found that maternal race can be a predictor of PFAS levels in children, with children born to black mothers showing lower PFAS levels than children born to white mothers. ${ }^{62-64}$ Additional research on the relationship between PFAS levels and maternal race and ethnicity is warranted to discern environmental exposure disparities, sources, and pathways.

Finally, this study evaluated changes in PFAS with gestational age at birth and pre-eclampsia or gestational hypertension. Previously, Mamsen and colleagues investigated placentas in the first through third trimester and identified five PFAS, including PFOS, PFOA, PFDA, PFNA, and PFUnA, that increased as gestational age increased. ${ }^{41,42}$ In the present study, none of the PFAS displayed a significant association between PFAS levels and gestational age. The differences between the Mamsen studies and the current study could be due to several factors. First, this high-risk cohort is enriched with placentas from preterm deliveries compared to the larger U.S. population. As a result, the placentas are likely physiologically distinct from term placentas, which may influence PFAS accumulation. ${ }^{65,66}$ Second, the current study investigated placentas from midsecond to third trimester pregnancies (18-41 weeks' gestation), while Mamsen et al. investigated placentas from the first to third trimesters. Third, our study cohort placentas were obtained from live births, while the Mamsen studies investigated PFAS in the placenta and fetal organs from intrauterine fetal demise (IUFD). In addition, in the present study, we observed no associations between pre-eclampsia or gestational hypertension and placental PFAS. Previous literature is divided on the subject of PFAS and pre-eclampsia with one study reporting no association, ${ }^{17}$ while other studies report associations for at least one PFAS, including PFOS, PFOA, PFNA, and PFBS. ${ }^{15,16,19,20}$ It is important to note that placental studies 
are limited to cross-sectional design and therefore hindered by the potential for reverse causality. ${ }^{67,68}$

While this study is among the first to examine PFAS placental levels in a U.S. cohort, it is not without limitations. Concentrations of PFAS quantified in the placenta are usually lower than matching fetal cord serum and maternal serum. ${ }^{40}$ This limitation often reduces the number of PFAS measured above the reporting limit and can impact the ability of researchers to identify associations between PFAS and potential risk factors; for example, PFUnA, in our study, was detected above the $\mathrm{RL}$ in $49 \%$ of placentas. Future studies will investigate PFAS in matching maternal serum from this cohort. Finally, the cohort used in this study comprises women at high risk for spontaneous preterm birth in North Carolina. As mentioned, the exposure levels and associations detected in this cohort are likely not generalizable to the broader U.S. population of pregnant women due to the geographical and obstetric characteristics of this cohort. ${ }^{69}$ Specifically, pathologies of the placenta may influence bioaccumulation of PFAS, and thus, reverse causality cannot be excluded with a cross-sectional study. Future research should investigate the associations between PFAS accumulation in the placenta and pregnancy complications in a more generalizable cohort.

For the 122 placentas investigated, similar PFOS and PFOA levels were observed in this U.S.-based cohort as compared to cohorts in China and Denmark. Maternal race/ethnicity was associated with significant differences in PFUnA levels. No significant associations between PFAS and fetal growth, gestational age, or hypertensive disorders of pregnancy were observed in the current study. Overall, this study expands on the limited data describing PFAS levels in the placenta and further highlights the need for biomonitoring of PFAS during pregnancy among women of child-bearing age.

\section{ASSOCIATED CONTENT}

\section{Supporting Information}

The Supporting Information is available free of charge at https://pubs.acs.org/doi/10.1021/acs.est.9b07102.

Additional materials and methods; cohort comparison to the National Vital Statistics Birth Data 2016 and 2017; all calculated RLs (File S1, XLSX); associations between each of PFAS levels measured in the placenta (Figure S1); Spearman correlation coefficients of the correlations between PFAS measured in the placenta (Table S1); comparisons of medians of PFAS levels by selected demographic variables (Table S2); comparisons of medians, IQR, and maximum and minimum values of PFAS levels by hypertensive disorders of pregnancy (Table S3); comparisons of medians, IQR, and maximum and minimum values of PFAS levels by fetal growth (Table S4); $\beta$ values, standard error, and associated $p$ values of crude linear regressions of PFAS level onto gestational age; $\beta$ values, standard error, and associated $p$ values of adjusted, multivariate linear regressions of PFAS level onto gestational age (Table S5); crude binomial regression models between gestational age and PFAS detection above RL for the seven PFAS detected below $\mathrm{RL}$ in approximately $50 \%$ or more of samples (Table S6); $\beta$ values, standard error, and associated $p$-values of crude linear regressions of PFAS level onto delivery date (Table S7); and NIST Standard Reference Material 1947 CoA compared to PFAS values obtained in this study (Table S8) (PDF)

\section{AUTHOR INFORMATION}

\section{Corresponding Author}

Rebecca C. Fry - Department of Environmental Sciences and Engineering, Gillings School of Global Public Health, Institute for Environmental Health Solutions, Gillings School of Global Public Health, and Curriculum in Toxicology, University of North Carolina at Chapel Hill, Chapel Hill, North Carolina 27516, United States; (1) orcid.org/0000-0003-0899-9018; Email: rfry@unc.edu

\section{Authors}

Jacqueline Bangma - Department of Environmental Sciences and Engineering, Gillings School of Global Public Health, University of North Carolina at Chapel Hill, Chapel Hill, North Carolina 27516, United States; (1) orcid.org/0000-0003-1512-173X

Lauren A. Eaves - Department of Environmental Sciences and Engineering, Gillings School of Global Public Health, University of North Carolina at Chapel Hill, Chapel Hill, North Carolina 27516, United States; 이이이.org/0000-0001-6056-923X

Kirsi Oldenburg - Department of Environmental Sciences and Engineering, Gillings School of Global Public Health, University of North Carolina at Chapel Hill, Chapel Hill, North Carolina 27516, United States

Jessica L. Reiner - Chemical Sciences Division, Hollings Marine Laboratory, National Institute of Standards and Technology, Charleston, South Carolina 29412, United States

Tracy Manuck - Department of Obstetrics and Gynecology, Division of Maternal Fetal Medicine, University of North Carolina-Chapel Hill, Chapel Hill, North Carolina 27599, United States; Institute for Environmental Health Solutions, Gillings School of Global Public Health, University of North Carolina at Chapel Hill, Chapel Hill, North Carolina 27599, United States

Complete contact information is available at: https://pubs.acs.org/10.1021/acs.est.9b07102

\section{Author Contributions}

T.M. and R.C.F. contributed equally to this work.

\section{Notes}

Certain commercial equipment or instruments are identified in the paper to adequately specify the experimental procedures. Such identification does not imply recommendations or endorsement by the NIST nor does it imply that the equipment or instruments are the best available for the purpose. The authors declare no competing financial interest.

\section{ACKNOWLEDGMENTS}

This research was funded in part by the NIH (T32-ES007018, R01-MD011609, and K24-ES031131) and the NC PFAST network.

\section{REFERENCES}

(1) KEMI. Occurrence and Use of Highly Fluorinated Substances and Alternatives; KEMI, Swedish Chemicals Agency Stockholm: Sweden, 2015.

(2) Eriksson, U.; Kärrman, A. World-wide indoor exposure to polyfluoroalkyl phosphate esters (PAPs) and other PFASs in household dust. Environ. Sci. Technol. 2015, 49, 14503-14511.

(3) Hu, X. C.; Andrews, D. Q.; Lindstrom, A. B.; Bruton, T. A.; Schaider, L. A.; Grandjean, P.; Lohmann, R.; Carignan, C. C.; Blum, A.; 
Balan, S. A.; Higgins, C. P.; Sunderland, E. M. Detection of Poly- and Perfluoroalkyl Substances (PFASs) in U.S. Drinking Water Linked to Industrial Sites, Military Fire Training Areas, and Wastewater Treatment Plants. Environ. Sci. Technol. Lett. 2016, No. 260.

(4) Hopkins, Z. R.; Sun, M.; DeWitt, J. C.; Knappe, D. R. Recently Detected Drinking Water Contaminants: GenX and Other Per-and Polyfluoroalkyl Ether Acids. J. - Am. Water Works Assoc. 2018, No. 107. (5) Haug, L. S.; Thomsen, C.; Brantsæter, A. L.; Kvalem, H. E.; Haugen, M.; Becher, G.; Alexander, J.; Meltzer, H. M.; Knutsen, H. K. Diet and particularly seafood are major sources of perfluorinated compounds in humans. Environ. Int. 2010, 36, 772-778.

(6) Tittlemier, S. A.; Pepper, K.; Seymour, C.; Moisey, J.; Bronson, R.; Cao, X.-L.; Dabeka, R. W. Dietary exposure of Canadians to perfluorinated carboxylates and perfluorooctane sulfonate via consumption of meat, fish, fast foods, and food items prepared in their packaging. J. Agric. Food Chem. 2007, 55, 3203-3210.

(7) Fromme, H.; Mosch, C.; Morovitz, M.; Alba-Alejandre, I.; Boehmer, S.; Kiranoglu, M.; Faber, F.; Hannibal, I.; GenzelBoroviczeny, O.; Koletzko, B.; Volkel, W. Pre- and postnatal exposure to perfluorinated compounds (PFCs). Environ. Sci. Technol. 2010, 44, $7123-7129$.

(8) Haug, L.; Huber, S.; Becher, G.; Thomsen, C. Characterisation of human exposure pathways to perfluorinated compounds-comparing exposure estimates with biomarkers of exposure. Environ. Int. 2011, 37, 687-693.

(9) Key, B.; Howell, R.; Criddle, C. Fluorinated Organics in the Biosphere. Environ. Sci. Technol. 1997, 31, No. 2445.

(10) Fromme, H.; Tittlemier, S.; Volkel, W.; Wilhelm, M.; Twardelle, D. Perfluorinated compounds-exposure assessment for the general population in Western countries. Int. J. Hyg. Environ. Health 2009, 212, 239-270.

(11) Kannan, K.; Corsolini, S.; Falandysz, J.; Fillmann, G.; Kumar, K.; Loganathan, B.; Mohd, M.; Olivero, J.; Van Wouwe, N.; Yang, J.; ALdoust, K. Perfluorooctanesulfonate and related fluorochemicals in human blood from several countries. Environ. Sci. Technol. 2004, 38, 4489-4495.

(12) US EPA. Drinking Water Advisory for Perfluorooctane Sulfonate (PFOS); United States Environmental Protection Agency: Washington, DC, 2016; pp 1-88.

(13) Calafat, A. M.; Wong, L. Y.; Kuklenyik, Z.; Reidy, J. A.; Needham, L. L. Polyfluoroalkyl chemicals in the U.S. population: data from the National Health and Nutrition Examination Survey (NHANES) 20032004 and comparisons with NHANES 1999-2000. Environ. Health Perspect. 2007, 115, 1596-1602.

(14) Emerging Contaminants and Federal Facility Contaminants of Concern. U.S. Environmental Protection Agency, 2019. https://www. epa.gov/fedfac/emerging-contaminants-and-federal-facilitycontaminants-concern.

(15) Huang, R.; Chen, Q.; Zhang, L.; Luo, K.; Chen, L.; Zhao, S.; Feng, L.; Zhang, J. Prenatal exposure to perfluoroalkyl and polyfluoroalkyl substances and the risk of hypertensive disorders of pregnancy. Environ. Health 2019, 18, 5.

(16) Wikström, S.; Lindh, C. H.; Shu, H.; Bornehag, C.-G. Early pregnancy serum levels of perfluoroalkyl substances and risk of preeclampsia in Swedish women. Sci. Rep. 2019, 9, No. 2045.

(17) Starling, A. P.; Engel, S. M.; Richardson, D. B.; Baird, D. D.; Haug, L. S.; Stuebe, A. M.; Klungsøyr, K.; Harmon, Q.; Becher, G.; Thomsen, C.; Sabaredzovic, A.; Eggesbø, M.; Hoppin, J. A.; Travlos, G. S.; Wilson, R. E.; Trogstad, L. I.; Magnus, P.; MP, L. Perfluoroalkyl substances during pregnancy and validated preeclampsia among nulliparous women in the Norwegian Mother and Child Cohort Study. Am. J. Epidemiol. 2014, 179, 824-833.

(18) Vestergaard, S.; Nielsen, F.; Andersson, A.-M.; Hjøllund, N. H.; Grandjean, P.; Andersen, H. R.; Jensen, T. K. Association between perfluorinated compounds and time to pregnancy in a prospective cohort of Danish couples attempting to conceive. Hum. Reprod. 2012, 27, 873-880.

(19) Savitz, D. A.; Stein, C. R.; Bartell, S. M.; Elston, B.; Gong, J.; Shin, H. M.; Wellenius, G. A. Perfluorooctanoic acid exposure and pregnancy outcome in a highly exposed community. Epidemiology 2012, 23, 386392.

(20) Stein, C. R.; Savitz, D. A.; Dougan, M. Serum levels of perfluorooctanoic acid and perfluorooctane sulfonate and pregnancy outcome. Am. J. Epidemiol. 2009, 170, 837-846.

(21) Jensen, T. K.; Andersen, L. B.; Kyhl, H. B.; Nielsen, F.; Christesen, H. T.; Grandjean, P. Association between Perfluorinated Compound Exposure and Miscarriage in Danish Pregnant Women. PLoS One 2015, 10, No. e0123496.

(22) Arbuckle, T. E.; Kubwabo, C.; Walker, M.; Davis, K.; Lalonde, K.; Kosarac, I.; Wen, S. W.; Arnold, D. L. Umbilical cord blood levels of perfluoroalkyl acids and polybrominated flame retardants. Int. J. Hyg. Environ. Health 2013, 216, 184-194.

(23) Fei, C.; McLaughlin, J.; Tarone, R.; J, O. Perfluorinated chemicals and fetal growth: a study within the Danish National Birth Cohort. Environ. Health Perspect. 2007, 115, 1677-1682.

(24) Lauritzen, H.; Larose, T.; Øien, T.; Sandanger, T.; Odland, J.; van de Bor, M.; Jacobsen, G. Maternal serum levels of perfluoroalkyl substances and organochlorines and indices of fetal growth: a Scandinavian case-cohort study. Pediatr. Res. 2017, 81, 33-42.

(25) Maisonet, M.; Terrell, M.; McGeehin, M.; Christensen, K.; Holmes, A.; Calafat, A.; Marcus, M. Maternal concentrations of polyfluoroalkyl compounds during pregnancy and fetal and postnatal growth in British girls. Environ. Health Perspect. 2012, 120, 1432-1437.

(26) Apelberg, B. J.; Witter, F. R.; Herbstman, J. B.; Calafat, A. M.; Halden, R. U.; Needham, L. L.; Goldman, L. R. Cord serum concentrations of perfluorooctane sulfonate (PFOS) and perfluorooctanoate (PFOA) in relation to weight and size at birth. Environ. Health Perspect. 2007, 115, 1670.

(27) Fei, C.; McLaughlin, J. K.; Tarone, R. E.; et al. Perfluorinated chemicals and fetal growth: a study within the Danish National Birth Cohort. Environ. Health Perspect. 2007, 115, 1677-1682.

(28) Liew, Z.; Ritz, B.; Bonefeld-Jorgensen, E. C.; Henriksen, T. B.; Nohr, E. A.; Bech, B. H.; Fei, C.; Bossi, R.; von Ehrenstein, O. S.; Streja, E.; Uldall, P.; Olsen, J. Prenatal exposure to perfluoroalkyl substances and the risk of congenital cerebral palsy in children. Am. J. Epidemiol. 2014, 180, 574-581.

(29) Ernst, A.; Brix, N.; Lauridsen, L.; Olsen, J.; Parner, E.; Liew, Z.; Olsen, L.; Ramlau-Hansen, C. Exposure to Perfluoroalkyl Substances during Fetal Life and Pubertal Development in Boys and Girls from the Danish National Birth Cohort. Environ. Health Perspect. 2019, 127, No. 017004.

(30) Braun, J.; Chen, A.; Romano, M.; Calafat, A.; Webster, G.; Yolton, K.; Lanphear, B. Prenatal perfluoroalkyl substance exposure and child adiposity at 8 years of age: The HOME study. Obesity 2015, 24, 231-237.

(31) Lau, C.; Thibodeaux, J. R.; Hanson, R. G.; Narotsky, M. G.; Rogers, J. M.; Lindstrom, A. B.; Strynar, M. J. Effects of perfluorooctanoic acid exposure during pregnancy in the mouse. Toxicol. Sci. 2006, 90, 510-518.

(32) Lau, C.; Butenhoff, J. L.; Rogers, J. M. The developmental toxicity of perfluoroalkyl acids and their derivatives. Toxicol. Appl. Pharmcol. 2004, 198, 231-241.

(33) Lau, C.; Thibodeaux, J. R.; Hanson, R. G.; Rogers, J. M.; Grey, B. E.; Stanton, M. E.; Butenhoff, J. L.; Stevenson, L. A. Exposure to perfluorooctane sulfonate during pregnancy in rat and mouse. II: postnatal evaluation. Toxicol. Sci. 2003, 74, 382-392.

(34) Tucker, D. K.; Macon, M. B.; Strynar, M. J.; Dagnino, S.; Andersen, E.; Fenton, S. E. The mammary gland is a sensitive pubertal target in CD-1 and C57Bl/6 mice following perinatal perfluorooctanoic acid (PFOA) exposure. Reprod. Toxicol. 2015, 54, 26-36.

(35) Lauritzen, H.; Larose, T.; Øien, T.; Odland, J.; van de Bor, M.; Jacobsen, G.; Sandanger, T. Factors Associated with Maternal Serum Levels of Perfluoroalkyl Substances and Organochlorines: A Descriptive Study of Parous Women in Norway and Sweden. PLoS One 2016, 11, No. e0166127.

(36) Sagiv, S. K.; Rifas-Shiman, S. L.; Webster, T. F.; Mora, A. M.; Harris, M. H.; Calafat, A. M.; Ye, X.; Gillman, M. W.; Oken, E. Sociodemographic and Perinatal Predictors of Early Pregnancy Per- 
and Polyfluoroalkyl Substance (PFAS) Concentrations. Environ. Sci. Technol. 2015, 49, 11849-11858.

(37) Glynn, A.; Berger, U.; Bignert, A.; Ullah, S.; Aune, M.; Lignell, S.; Darnerud, P. O. Perfluorinated alkyl acids in blood serum from primiparous women in Sweden: serial sampling during pregnancy and nursing, and temporal trends 1996-2010. Environ. Sci. Technol. 2012, 46, 9071-9079.

(38) Porpora, M. G.; Lucchini, R.; Abballe, A.; Ingelido, A. M.; Valentini, S.; Fuggetta, E.; Cardi, V.; Ticino, A.; Marra, V.; Fulgenzi, A. R. Placental transfer of persistent organic pollutants: a preliminary study on mother-newborn pairs. Int. J. Environ. Res. Public Health 2013, 10, 699-711.

(39) Gao, K.; Zhuang, T.; Liu, X.; Fu, J.; Zhang, J.; Fu, J.; Wang, L.; Zhang, A.; Liang, Y.; Song, M.; Jiang, G. Prenatal Exposure to Per- and Polyfluoroalkyl Substances (PFASs) and Association between the Placental Transfer Efficiencies and Dissociation Constant of Serum Proteins-PFAS Complexes. Environ. Sci. Technol. 2019, No. 6529.

(40) Chen, F.; Yin, S.; Kelly, B. C.; Liu, W. Chlorinated polyfluoroalkyl ether sulfonic acids in matched maternal, cord, and placenta samples: a study of transplacental transfer. Environ. Sci. Technol. 2017, 51, 6387-6394.

(41) Mamsen, L. S.; Björvang, R. D.; Mucs, D.; Vinnars, M.-T.; Papadogiannakis, N.; Lindh, C. H.; Andersen, C. Y.; Damdimopoulou, P. Concentrations of perfluoroalkyl substances (PFASs) in human embryonic and fetal organs from first, second, and third trimester pregnancies. Environ. Int. 2019, 124, 482-492.

(42) Mamsen, L. S.; Jönsson, B. A.; Lindh, C. H.; Olesen, R. H.; Larsen, A.; Ernst, E.; Kelsey, T. W.; Andersen, C. Y. Concentration of perfluorinated compounds and cotinine in human foetal organs, placenta, and maternal plasma. Sci. Total Environ. 2017, 596, 97-105. (43) Monroy, R.; Morrison, K.; Teo, K.; Atkinson, S.; Kubwabo, C.; Stewart, B.; Foster, W. G. Serum levels of perfluoroalkyl compounds in human maternal and umbilical cord blood samples. Environ. Res. 2008, $108,56-62$.

(44) Calafat, A. M.; Kuklenyik, Z.; Reidy, J. A.; Caudill, S. P.; Tully, J. S.; Needham, L. L. Serum concentrations of 11 polyfluoroalkyl compounds in the US population: data from the National Health and Nutrition Examination Survey (NHANES) 1999-2000. Environ. Sci. Technol. 2007, 41, 2237-2242.

(45) Sun, M.; Arevalo, E.; Strynar, M.; Lindstrom, A.; Richardson, M.; Kearns, B.; Pickett, A.; Smith, C.; Knappe, D. R. Legacy and emerging perfluoroalkyl substances are important drinking water contaminants in the Cape Fear River Watershed of North Carolina. Environ. Sci. Technol. Lett. 2016, 3, 415-419.

(46) Manuck, T. A.; Smeester, L.; Martin, E. M.; Tomlinson, M. S.; Smith, C.; Varner, M. W.; Fry, R. C. Epigenetic Regulation of the Nitric Oxide Pathway, 17- $\alpha$ Hydroxyprogesterone Caproate, and Recurrent Preterm Birth. Am. J. Perinatol. 2018, 35, 721-728.

(47) ACOG. Practice Bulletin No. 202: Gestational Hypertension and Preeclampsia. Am. J. Obstet. Gynecol. 2019, 133, e1-e25.

(48) Bangma, J. T.; Reiner, J. L.; Lowers, R. H.; Cantu, T. M.; Scott, J.; Korte, J. E.; Scheidt, D. M.; McDonough, C.; Tucker, J.; Back, B. Perfluorinated alkyl acids and fecundity assessment in striped mullet ( Mugil cephalus) at Merritt Island national wildlife refuge. Sci. Total Environ. 2018, 619, 740-747.

(49) Reiner, J. L.; O’Connell, S. G.; Moors, A. J.; Kucklick, J. R.; Becker, P. R.; Keller, J. M. Spatial and temporal trends of perfluorinated compounds in beluga whales (Delphinapterus leucas) from Alaska. Environ. Sci. Technol. 2011, 45, 8129-8136.

(50) Kurtz, A. E.; Reiner, J. L.; West, K. L.; Jensen, B. A. Perfluorinated Alkyl Acids in Hawaiian Cetaceans and Potential Biomarkers of Effect: Peroxisome Proliferator-Activated Receptor Alpha and Cytochrome P450 4A. Environ. Sci. Technol. 2019, 53, No. 2830.

(51) Keller, J. M.; Kannan, K.; Taniyasu, S.; Yamashita, N.; Day, R. D.; Arendt, M. D.; Segars, A. L.; Kucklick, J. R. Perfluorinated Compounds in the Plasma of Loggerhead and Kemp's Ridley Sea Turtles from the Southeastern Coast of the United States. Environ. Sci. Technol. 2005, 39, 9101-9108.
(52) Olsen, I. E.; Groveman, S. A.; Lawson, M. L.; Clark, R. H.; Zemel, B. S. New intrauterine growth curves based on United States data. Pediatrics 2010, 125, e214-24.

(53) Hamilton, B. E.; Osterman, M. J.; Driscoll, A. K.; Rossen, L. M. Births: Provisional Data for 2017; National Vital Statistics Report, 2018; pp 14-22.

(54) Martin, J. A.; Hamilton, B. E.; Osterman, M. J. K.; Driscoll, A. K.; Drake, P. Births: Final Data for 2016; National Vital Statistics Report, 2018; pp 1-55.

(55) Sagiv, S. K.; Rifas-Shiman, S. L.; Fleisch, A. F.; Webster, T. F.; Calafat, A. M.; Ye, X.; Gillman, M. W.; Oken, E. Early-Pregnancy Plasma Concentrations of Perfluoroalkyl Substances and Birth Outcomes in Project Viva: Confounded by Pregnancy Hemodynamics? Am. J. Epidemiol. 2018, 187, 793-802.

(56) Pérez, F.; Nadal, M.; Navarro-Ortega, A.; Fàbrega, F.; Domingo, J. L.; Barceló, D.; Farré, M. Accumulation of perfluoroalkyl substances in human tissues. Environ. Int. 2013, 59, 354-362.

(57) Centers for Disease Control and Prevention. Fourth National Report on Human Exposure to Environmental Chemicals; National Center for Environmental Health (U.S.) Division of Laboratory Sciences: Atlanta, Georgia, 2019; pp 1-866.

(58) US EPA. Drinking Water Health Advisory for Perfluorooctane Sulfonate (PFOS); U.S. Environmental Protection Agency Office of Water, 2016; pp 1-88.

(59) Christensen, K.; Raymond, M.; Blackowicz, M.; Liu, Y.; Thompson, B.; Anderson, H.; Turyk, M. Perfluoroalkyl substances and fish consumption. Environ. Res. 2017, 154, 145-151.

(60) Park, S.; Peng, Q.; Ding, N.; Mukherjee, B.; Harlow, S. Determinants of per- and polyfluoroalkyl substances (PFAS) in midlife women: Evidence of racial/ethnic and geographic differences in PFAS exposure. Environ. Res. 2019, 175, 186-199.

(61) Barton, K. E.; Starling, A. P.; Higgins, C. P.; McDonough, C. A.; Calafat, A. M.; Adgate, J. L. Sociodemographic and behavioral determinants of serum concentrations of per-and polyfluoroalkyl substances in a community highly exposed to aqueous film-forming foam contaminants in drinking water. Int. J. Hyg. Environ. Health 2020, 223, 256-266.

(62) Kato, K.; Wong, L.; Chen, A.; Dunbar, C.; Webster, G.; Lanphear, B.; Calafat, A. Changes in Serum Concentrations of Maternal Poly- and Perfluoroalkyl Substances over the Course of Pregnancy and Predictors of Exposure in a Multiethnic Cohort of Cincinnati, Ohio Pregnant Women during 2003-2006. Environ. Sci. Technol. 2014, 48, 9600-9608.

(63) Kingsley, S.; Eliot, M.; Kelsey, K.; Calafat, A.; Ehrlich, S.; Lanphear, B.; Chen, A.; Braun, J. Variability and predictors of serum perfluoroalkyl substance concentrations during pregnancy and early childhood. Environ. Res. 2018, 165, 247-257.

(64) Harris, M.; Rifas-Shiman, S.; Calafat, A.; Ye, X.; Mora, A.; Webster, T.; Oken, E.; Sagiv, S. Predictors of Per- and Polyfluoroalkyl Substance (PFAS) Plasma Concentrations in 6-10 Year Old American Children. Environ. Sci. Technol. 2017, 51, 5193-5204.

(65) Doğan, K.; Salihoglu, O.; Sever, N.; Tombul, T.; Sari, E.; Yaşar, L. Do placental histopathologic characteristics differ with gestational ages in preterm and term deliveries? Fetal Pediatr. Pathol. 2015, 34, 365374.

(66) Paquette, A. G.; Brockway, H. M.; Price, N. D.; Muglia, L. J. Comparative transcriptomic analysis of human placentae at term and preterm delivery. Biol. Reprod. 2018, 98, 89-101.

(67) Savitz, D. A.; Wellenius, G. A. Invited commentary: exposure biomarkers indicate more than just exposure. Am. J. Epidemiol. 2018, $187,803-805$.

(68) Dhingra, R.; Winquist, A.; Darrow, L. A.; Klein, M.; Steenland, K. A study of reverse causation: Examining the associations of perfluorooctanoic acid serum levels with two outcomes. Environ. Health Perspect. 2017, 125, 416-421.

(69) Kleinbaum, D. G.; Morgenstern, H.; Kupper, L. L. Selection bias in epidemiologic studies. Am. J. Epidemiol. 1981, 113, 452-463. 